\title{
The Technology-Mediated Reflection Model: Barriers and Assistance in Data-Driven Reflection
}

\author{
Marit Bentvelzen \\ Utrecht University \\ Utrecht, the Netherlands \\ m.bentvelzen@uu.nl
}

\author{
Jasmin Niess \\ University of Bremen \\ Bremen, Germany \\ niessj@uni-bremen.de
}

\author{
Paweł W. Woźniak \\ Utrecht University \\ Utrecht, the Netherlands \\ p.w.wozniak@uu.nl
}

\begin{abstract}
Current personal informatics models consider reflection as an important stage in users' journeys with trackers. However, these models describe reflection from a meta perspective and it remains unclear what this stage entails. To design interactive technologies that support reflection, we need a more thorough understanding of how people reflect on their personal data in practice. To that end, we conducted semi-structured interviews with users of fitness trackers and an online survey to study practices in reflecting on fitness data. Our results show that users reported reflecting on data despite lacking reflection support from their tracking technology. Based on our results, we introduce the Technology-Mediated Reflection Model, which describes conditions and barriers for reflection on personal data. Our model consists of the temporal and conceptual cycles of reflection and helps designers identify the possible barriers a user might face when using a system for reflection.
\end{abstract}

\section{CCS CONCEPTS}

- Human-centered computing $\rightarrow$ Empirical studies in ubiquitous and mobile computing.

\section{KEYWORDS}

personal informatics, reflection, tracking, fitness trackers, construal

ACM Reference Format:

Marit Bentvelzen, Jasmin Niess, and Paweł W. Woźniak. 2021. The TechnologyMediated Reflection Model: Barriers and Assistance in Data-Driven Reflection. In CHI Conference on Human Factors in Computing Systems (CHI '21), May 8-13, 2021, Yokohama, Japan. ACM, New York, NY, USA, 12 pages. https://doi.org/10.1145/3411764.3445505

\section{INTRODUCTION}

Reflection is considered to be an important part of how people use personal informatics systems [12,23]. By reflecting on personal data, a user can come to an understanding of patterns and trends, which can lead to more knowledge about oneself. However, previous research has shown that users accumulate long term personal data as a by-product of short term tracking [38]. In other words,

Permission to make digital or hard copies of all or part of this work for personal or classroom use is granted without fee provided that copies are not made or distributed for profit or commercial advantage and that copies bear this notice and the full citation on the first page. Copyrights for components of this work owned by others than the author(s) must be honored. Abstracting with credit is permitted. To copy otherwise, or republish, to post on servers or to redistribute to lists, requires prior specific permission and/or a fee. Request permissions from permissions@acm.org.

CHI '21, May 8-13, 2021, Yokohama, Japan

(C) 2021 Copyright held by the owner/author(s). Publication rights licensed to ACM. ACM ISBN 978-1-4503-8096-6/21/05 ...\$15.00

https://doi.org/10.1145/3411764.3445505 people do not use their activity data for analysis of trends or patterns, just for short-term monitoring or feedback. A possible cause for this is that current systems often do not support users in reflecting on their personal data [7-9, 22, 24], which might explain the increased interest in $\mathrm{HCI}$ to design for reflection $[3,25]$. Yet, to be able to design for reflection, we first need to have a more thorough understanding of what reflection entails for users of personal informatics systems. Current models for personal informatics, such as the Stage-Based Model of Personal Informatics Systems [23] and the Lived Informatics Model of Personal Informatics [12], describe reflection from a meta-perspective. These models form a key level of understanding in personal informatics. A recent literature review by Epstein et al. [10] remarked that publications about personal informatics systems often use a model as their theoretical framing. As such, the existing personal informatics models fuel the field and future interventions. Current models [12,23] offer a high-level overview of the process people go through in using personal informatics systems, but give us no insight into how reflection on personal data occurs in practice.

Thus, a better understanding of how people reflect on their personal data is needed. To explore this, we conducted interviews and an online survey with users who reported having fitness tracker routines. Through these interviews we established an account of the way in which people use their tracker's data for reflection. The results of our study show that reflection does occur, but users attribute that more to their reflective nature than to the fitness tracker's support for reflection. Our participants mentioned several barriers for reflection, resulting in users building their own, additional systems or maintaining spreadsheets to be able to reflect on their data. The interview study was followed by a survey where we inquired about the barriers and opportunities mentioned in the interviews in a larger group of users. Based on the gathered data, we propose the Technology-Mediated Reflection Model. Our model describes how two cycles, a temporal cycle and a conceptual cycle contribute to facilitated reflection on tracker data. The cycles illustrate how systems support or impede reflection. Our model is intended to serve as a sub-model for existing personal informatics models and it can be used to better understand existing systems as well as serve as an inspiration for future personal informatics designs.

This paper contributes the following: (1) a qualitative study of the practices in reflection on data accumulated by fitness trackers consisting of 20 interviews; (2) a survey of the conceptual and temporal aspects of reflection among personal informatics users; (3) an account of the users' current practices with reflecting on personal data; and (4) the Technology-Mediated Reflection Model (TMRM), which describes conditions and barriers for reflection. 
In this work, we first review related literature in reflection and personal informatics. We then present the method and findings from our interviews and a survey. Next, we use the gathered data to introduce the Technology-Mediated Reflection Model (TMRM). We conclude with a discussion and directions for future research.

\section{RELATED WORK}

In this section, we first report on the conceptualisation of reflection in the field of HCI. We then discuss how reflection is manifested as a design goal in interactive technologies. Next, we report on user's experiences of personal informatics journeys. Finally, we discuss past accounts of personal informatics experiences involving reflection

\subsection{Reflection in HCI}

The HCI field invested considerable effort in building an understanding about reflection. HCI work is highly influenced by Schön's framing of reflection [2, 36]. A systematic review conducted by Baumer [2] shows that $70 \%$ of HCI papers (that explicitly define reflection) use Schön's notion of reflection-in-action or reflectionon-action. Reflection-in-action takes place during action, implying that a person is reflecting on their actions while doing them [35] According to Schön [26], reflection-in-action does not occur during actions that go according to plan, it only occurs in situations where the action leads to unexpected consequences. In contrast, reflection-on-action occurs after an action has finished. Reflectionon-action is the activity of reconstructing an experience, based on our memories of the event. It is an effort of stepping back into the experience, retrieving our memories and organising these fragmented parts with the aim of understanding what has happened and drawing lessons from the experience. However, as Slovak et al. [36] remarked, Schön's framework does not directly address how technology can support reflective processes, leading to a gap in our understanding of how reflection can be facilitated by technology. To address this gap, Slovak et al. adopt Schön's notion of the reflective practicum. The goal of the reflective practicum is to structure a learning process by offering a safe environment in which students learn through scaffolded learning experiences. Their work aims to create the 'right experiences', consisting of three components (explicit, social and personal) that reinforce each other in scaffolding reflection. Our work aims to continue the inquiry into the practices of technology supported reflection. More specifically, we investigate if and how current concepts of reflection in HCI can be used to understand reflection in personal informatics.

\subsection{Systems That Support Reflection}

A wide variety of systems was designed with reflection as the declared design goal. Such systems are often interpreted as more ethical successors to an earlier trend focused on persuasive technology [10]. Often, personal informatics systems aspired to enhance reflection among users by visualising past data [1, 6, 13, 30-32]. For instance, Trackly [1] was a mobile app that helped patients with Multiple Sclerosis to manually track their symptoms and to reflect on the collected data through visualisations. Using Trackly led to introspection among participants, as well as feelings of empowerment in handling their disease.
Concurrently, personal informatics systems have been critiqued in the past for not actively encouraging reflection [7, 22]. As noted by Baumer [2], these systems carry an implicit assumption that by showing a user visualisations of their past data for the purpose of reflection, that reflection will occur. However, as also noted by Slovak [36], this conflicts with reflection theories that underscore the importance of encouraging reflection, seeing that it often does not occur automatically. The literature also shows examples of technologies that adopt an active role in the encouragement of reflection. Isaacs et al. [19] designed a mobile app for recording everyday experiences and reflecting on them later. The app prompts old entries to users and lets them re-rate their current happiness regarding the memory. Other systems aimed to encourage reflection through asking reflective questions. Kocielnik et al. [21] designed Robota, a chatbot with voice interaction. Robota stimulated reflection and self-learning in the workplace by asking questions and chatting. A similar approach was used by Jung et al. [20], who designed a conversational agent that supported children in reflecting during designing and building mechatronics systems. The agent asked open-ended questions that stimulated a dialogue between a child and the artefact while building the system.

These examples are illustrative for the ongoing design efforts for technologies facilitating reflection, both in the field of personal informatics and broader HCI. These technologies use a wide spectrum of features to encourage reflection. Yet there is a gap in our understanding of how these different qualities of interactive technologies contribute to reflection beyond specific systems. Thus, it remains unclear how a system can help users enact reflection.

\subsection{Modeling the Personal Informatics Journey}

In recent years, several models were proposed that describe the user's journey in using personal informatics systems. Li's StageBased Model of Personal Informatics Systems [23] described this journey through five stages: preparation, collection, integration, reflection and action. Subsequently, Epstein et al. proposed the Lived Informatics Model of Personal Informatics [12], which is an extension of Li's model. Several stages have been added, such as deciding to track, selecting tools, and tracking and acting as an ongoing process of collection, integration and tracking. Later, Niess \& Wozniak [28] explained phenomena related to goal setting and placed them in the framework of Epstein et al.'s model. A common feature of the three models above is that they all feature a reflection phase and this phase is ascribed significant importance. Reflection is seen as a key enabler for positive experiences in one's personal informatics journey. Given the importance of reflection for the overall process, a deeper understanding of personal informatics can be built through studying the details of that phase. It remains a challenge to understand how and under what conditions users enter and exit the reflection phase. Consequently, there is a need for examining the user practices within the reflection phase of personal informatics journeys. While a number of models have been built that help us understand personal informatics journeys, these models do not account for the ways in which users interpret and understand data about themselves. 


\subsection{Interpreting Data in Personal Informatics}

Another facet of personal informatics research is understanding how users conceptualise and interpret their personal informatics experiences. For instance, Gouveia et al. [16] found that users often struggled to contextualise minimalist feedback produced by trackers and project the information onto their overall perception of fitness. The need for understanding fitness on an abstract level was further exemplified by Carrington et al. [5] who studied the experiences of wheelchair athletes. These users were dissatisfied because of the conceptual mismatch between the features commercial fitness trackers offered and their need to monitor their fitness in a meaningful way. Further, Murnane et al. [27] showed how different social stakeholders involved in the life of persons with serious mental illnesses who used trackers used tracking at different levels of abstraction.

On another note, Niess and Wozniak [28] found that users often aimed to relate qualitative goals to quantitative goals, thus operationalising numbers produced by tracking systems into higherlevel concepts that matched their personal fitness goals. Further, Niess and Wozniak [28] emphasised that personal informatics goals evolved over time. This view is also supported by Suh and Hsieh [37]. They explored how motivations for activities that would happen in the near or distant future (e.g. near or distant goals) could be addressed in technologies for behavioural change. They applied construal level theory (CLT) as an understanding lens for their work. Construal level theory (CLT), introduced by Trope et al. [39] describes the interplay between the level of construal and psychological distance. CLT differentiates between two levels of mental representation (high construal/low construal). In other words, one can think about a situation in abstract or concrete terms. For instance, 'going for a walk in the park' can be construed as: 'spending some time in nature to unwind and relax' (high construal, i.e. more abstract) or 'walking 10000 steps' (low construal, i.e. more concrete). Further, CLT postulates that the way people construe aspects of their life (e.g. how they think about events, objects, people) is connected to psychological distance. The more (psychologically) distant a certain situation is the more abstract it will be thought of (i.e. high construal), and the more (psychologically) closer a certain situation is the more concrete it will be thought of (i.e. low construal). Using the aforementioned example of the walk in the park, a higher level of construal induces a focus on the 'why' of the activity (i.e. 'spending some time in nature to unwind and relax'), whereas a lower construal level induces a focus on the 'how' of the activity (i.e. 'walking 10000 steps').

The works outlined above encompass a variety of approaches to build an understanding about the ways users conceptualise their personal informatics experiences. However, modern trackers still struggle with presenting information to users at the right level of abstraction $[16,28]$. Our work aims to understand how trackers can help users effectively understand and reflect about their tracking data and relate it to abstract concepts that represent their values and desires.

\section{METHOD}

This study inquires how the reflection phase in personal informatics experiences is enacted in the everyday lives of users. To that end, we conducted a mixed-method inquiry. We conducted a set of in-depth interviews and an online survey. This enabled us to gather both in-depth accounts of qualitative experiences in reflection tracking as well as quantitatively examining trends in how a broader sample of everyday users reflect on personal informatics data. The survey enabled us to examine if the qualitative insights from the interviews translated to a larger group of users. Next, we present the details of our data collection and analysis methods. We then show how the gathered data contributed to building the TMRM.

\subsection{Interviews}

We conducted semi-structured interviews with fitness tracker users. A number of past studies of personal informatics used interviews as a data source, e.g. [12, 28]. Further, we chose the interview format as it enabled us to address specifically the reflection phase of the tracking experience. Personal, in-depth interviews allowed charting the users' understanding of reflection and their practices connected with reflecting. Interviews are also possible to conduct over a distance, which was required when the study was conducted, i.e. April to June 2020.

3.1.1 Participants. We used our social networks combined with snowball sampling to recruit $n=20$ participants. We did not use strict participation criteria, and included all users with an intention to use tracking technology on a long-term basis. The participants were aged 21-53 years, $M=32.65, S D=9.39$. Twelve interviewees identified as male and eight as female. All participants were residents of the European Union and were interviewed in their native language or English. We used Skype and Microsoft Teams to conduct the interviews and record audio. The participants were asked for consent for recording before the interviews and informed that they are allowed to terminate the interview at any time. We provided shopping vouchers for EUR 10 as remuneration. Table 1 presents details about the participants.

3.1.2 Interview Protocol. The interview started with basic demographic data collection and obtaining consent for recording. We then inquired about the user's tracking history and the reasons behind using trackers. Next, we asked questions about what data they collected and when they reviewed the data ${ }^{1}$. We used contextual laddering [18] to gather detailed data about when data review took place and what tools were used to facilitate reflection. Having established what the users' routines were in reviewing data, we explicitly asked about their understanding of reflection. We inquired about when they felt they were reflecting, what prompted them to reflect and how reflection made them feel. The interview ended with a discussion of the impact of reflection on lifestyle decisions.

3.1.3 Data Analysis. We recorded audio throughout the interviews. In total, we collected 9 hours and 35 minutes of audio. An interview lasted 28 minutes on average, $S D=8.59$ minutes. All interviews were transcribed verbatim. We imported the transcripts into the Atlas.ti analysis software for further analysis. To build an inductive understanding of user practices and behaviours in the reflection phase of personal informatics, we applied an iterative analysis process [29]. As a starting point, two authors coded a representative

${ }^{1}$ The full protocol is included in the supplementary material. 
Table 1: An overview of the participants in the interview study. The tracking experience reported in the table comprises all periods of tracking and excludes possible lapses. Participants marked with an asterisk $\left({ }^{*}\right)$ reported lapses in their tracking experience.

\begin{tabular}{lllllll}
\hline PID & Gender & Age & Profession & Brand & Model & Tracking duration \\
\hline P1* & Male & 35 & Teacher & Polar & M200 & 2 years \\
P2 & Male & 24 & Student & Xiaomi & MiBand 3 & 1 year \\
P3 & Female & 47 & Teacher & Fitbit & Alta HR & 1 year \\
P4 & Male & 20 & Student & Fitbit & Charge 3 & 1 year \\
P5 & Female & 47 & Teacher & Fitbit & Alta HR & 3 years \\
P6 & Male & 38 & Teacher & Garmin & Forerunner 735XT & 5 years \\
P7 & Male & 53 & Entrepreneur & Garmin & Forerunner 735XT & 10 years \\
P8 & Male & 26 & Student & Huawei & Band 3 Pro & 2 years \\
P9 & Female & 21 & Student & TomTom & Spark 3 & 1 year 6 mo \\
P10* & Female & 23 & Student & WHOOP & 3.0 & 9 mo \\
P11 & Female & 26 & Project leader & Apple & Watch 4 & 1 year \\
P12 & Male & 39 & ICT manager & Garmin & Forerunner 735XT & 3 years \\
P13* & Female & 42 & Teacher & Fitbit & Charge 3 & 4 years \\
P14 & Male & 23 & Student & Garmin & Forerunner 235 & 2 years \\
P1* & Male & 32 & Fitness instructor & WHOOP & 3.0 & 3 years \\
P16 & Female & 28 & Lawyer & Apple & Watch 4 & 1 year 6 mo \\
P17 & Male & 35 & PE teacher & TomTom & Touch & 4 years \\
P18 & Male & 29 & Marketer & WHOOP & 3.0 & 2 months \\
P19 & Female & 33 & Kindergarten teacher & Fitbit & Charge 3 & 1 year 6 mo \\
P20* & Male & 32 & Telecommunications engineer & Fitbit & Charge 2 & 4 years \\
\hline
\end{tabular}

sample of $10 \%$ of the data using open coding in line with Blandford et al. [4]. Through a set of iterative discussion rounds we established an initial coding tree, which was then used by the first author to code the remaining material. Subsequently, we used thematic analysis combined with affinity diagramming [4], to gain a more abstract overview of the data. Finally, we applied axial coding [33] combined with iterative discussions to refine our understanding of the data. At this stage, we identified the concepts of abstractness and temporality (which serve as the core of our model). To investigate them in more detail, we returned to the data and recoded the quotations within those themes to increase granularity within the themes. Using the re-coded data, we conducted a second round of axial coding and constructed the model through constant comparison.

\subsection{Survey}

To increase the ecological validity of our inquiry, we conducted an online survey based on the results from our qualitative inquiry. This enabled us to additionally validate our findings within the identified concepts. Further, by using an online survey, we could reach out to a more diverse and larger user sample. The full questionnaire and data set is available as auxiliary material.

3.2.1 Participants. We used Amazon Mechanical Turk to recruit $n=60$ participants for the study. Participants were required to have at least 1000 completed tasks with an acceptance rate of at least $95 \%$. To complete the survey, participants needed to declare that they owned and actively used a tracking technology and review data generated by it. The participants were aged 19-59 years, $M=32.14$,
$S D=8.09$ and resided primarily in the US, Canada, United Kingdom and the European Union. Forty-seven participants identified as male and twenty-one as female. The participants received USD 1 for completing the survey which lasted on average 4.27 minutes, $S D=4.27$ minutes. The majority of the survey participants reported that they were satisfied with their tracker, as shown in Figure 1.

3.2.2 Survey Content. Our online Qualtrics survey further investigated reflection practices that were identified in the interviews. The survey contained questions about the users' frequency of looking at their tracker data, their reflecting practices, and their satisfaction in using the device. Further, we specifically explored the temporal dimension of reflection on personal data through questions and comments such as 'When you look at your data, what is the time period you look at?', 'Reflecting on my data happens spontaneously' and 'I plan when to look back on my data'. Finally, the survey asked questions regarding the relation between the tracker's abilities and the user's needs: 'The information provided by the tracker matches what I want to achieve with the tracker' and 'I use additional tools (e.g. a spreadsheet) to reflect on my data'.

\section{THE TECHNOLOGY-MEDIATED REFLECTION MODEL}

The Technology-Mediated Reflection Model (TMRM) describes user behaviours and practices in the reflection phase of a personal informatics journey. The model shows how users enter, exit and stay in the reflection phase. The model is comprised of two cycles; the temporal cycle and the conceptual cycle. These cycles show how 


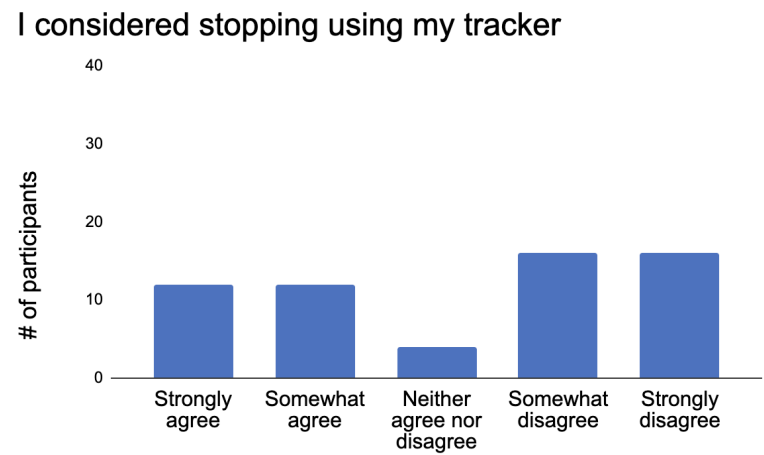

(a)

I am satisfied with my tracking experience

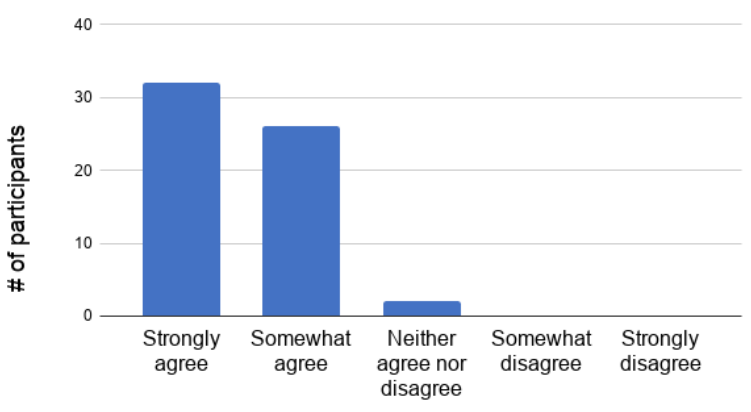

(b)

Figure 1: Considering lapsing (a) and overall tracker satisfaction (b) reported in our survey. Some survey participants considered whether to stop using their tracker, however most were very satisfied with their tracking experience.

users' needs and perspectives evolve throughout their engagement with their tracker.

The key concept behind the TMRM is facilitated reflection-a state where the user perceives that they receive adequate support for reflection from their tracking technology of choice, i.e. the features of the tracking technology are aligned with the current conceptual and temporal needs of the user. Our findings show that this state is temporary-reflection in personal informatics is a dynamic process in which the users constantly adapt their tracking experience to their evolving needs. An adapted tracking experience which facilitates effective reflection always happens in context and often leads to re-framing one's needs which in turn calls for adapting the tracking experience again. Thus, constant adaptation for alignment becomes a cyclic process. The TMRM describes how users desire different perspectives on their data and how these needs may change over time. The inability to effectively access data that provides the desired conceptual or temporal perspective will negatively impact reflection. Users will then, if possible, adapt their tracking experience and, if they have the means to implement the perspective shift, they are likely to re-enter facilitated reflection. If certain boundary conditions are unmet, the user may leave the reflection phase.
Our model operationalises the cyclic experience of reflection into two cycles: the temporal and the conceptual cycle. The temporal cycle describes how the users' perceptions of time evolve in a personal informatics experience. This cycle has two sub-cycles: temporal perspective and temporal frequency. The temporal perspective cycle describes the changes in the period of time, on which the users would like to reflect. The frequency cycle specifies when reflection happens, how often it happens, and how long it lasts. The conceptual cycle in the TMRM focuses on the levels of abstraction at which users interface with their tracking data. In this cycle, users constantly interpret and operationalise data in a reflection process, linking the feedback they receive from their tracker to their lived experience.

The starting point of the TMRM is the selection of tracking technology. Here, our model connects directly to Epstein et al.'s [12] model. The selection can either involve minimal effort (receiving the tracker as a gift) or an extensive comparison of tools. The selection of tools can depend on several things, such as features, aesthetics, budget and convenience [12]. Here, the TMRM describes what criteria are used during the selection phase. In our model, users select tracking technologies in order to achieve a conceptual match between the concept of what is to be tracked offered by the tracker and their own perception of the benefits of the tracker.

Figure 2 shows the different parts of the model. Below, we discuss each element in the TMRM in detail and show how these concepts are based on the data gathered in our study.

\section{BUILDING THE TMRM}

This section illustrates how the TMRM is built on the data gathered in our two studies. We show how the model describes the experiences related to reflection reported by the participants in our study.

\subsection{Selection of Tracking Technology}

We observed that multiple participants chose their tracker based on the device being marketed as specific to a sport which they practice. Active triathletes preferred a triathlon-specific Garmin Forerunner $735 \mathrm{XT}^{2}$ and those practising crossfit used the $\mathrm{WHOOP}^{3}$, which is marketed specifically for crossfit. One user described how their choice of tracker was dictated by its perceived conceptual suitability to their favourite sport:

Well, I actually bought this watch because it's a good fit for triathlons. It can track data for all the swimming, cycling and running, and more precisely, the combination of all three, that's a special [triathlon] function [on the tracker]. (P6)

Other users had diverging priorities when choosing their fitness trackers. All participants were aware of the qualities which they desired to see in their fitness tracker. These qualities ranged from pragmatic concerns, e.g. form factor to abstract concepts like aesthetics. An overarching concept was ensuring that the tracker provided an understanding of the desired outcome of using it. One user, a self-declared Apple fan girl commented on her concept of a fitness tracker as a question of identity:

\footnotetext{
${ }^{2}$ https://buy.garmin.com/en-US/US/p/541225/pn/010-01614-00

${ }^{3}$ https://www.whoop.com/membership/
} 


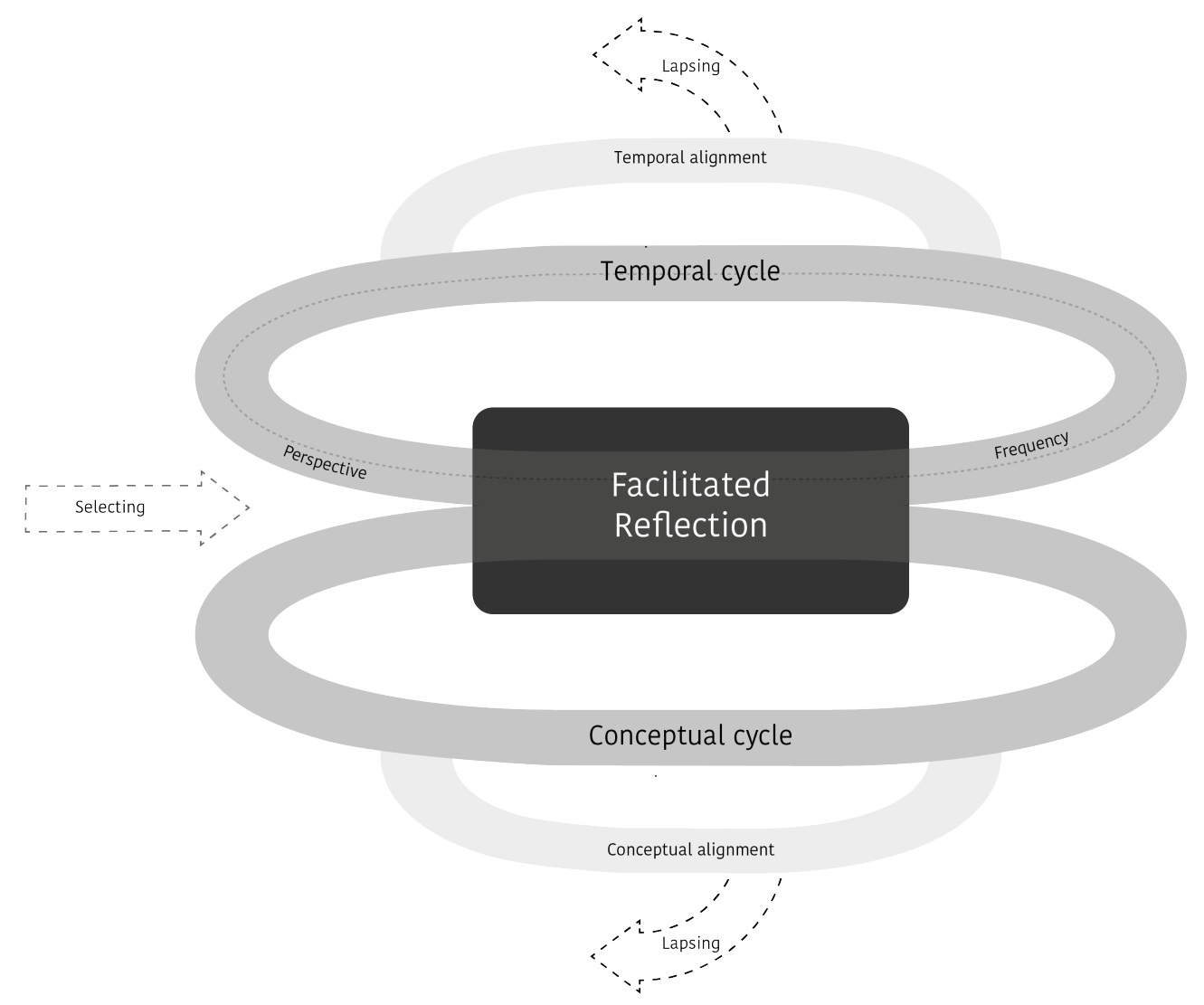

Figure 2: The Technology-Mediated Reflection Model. In our model, reflection happens in two cycles: temporal and conceptual. Users can customise and/or change tracker experiences to align them to their needs in the alignment phases. The dashed arrows show exit an entry point in the context of Epstein et al.'s [12] work.

It's not like I'm such a fit girl. It's not like I run or something, which is what most people use it [the fitness tracker] for. I'm just an Apple fan. (P16)

\subsection{Temporal Cycle}

Time and manipulating time was a strong theme in the gathered data. Tracker users reported that they often changed the time period that interested them in exploring their data. When reflection happens during the day also underwent constant changes.

5.2.1 Perspective. Our data analysis showed that users reflected upon a large spectrum of time intervals in their tracking practice, ranging from being only interested in data from a single day to prioritising year-to-year analysis. The online survey showed that there was variety in the preferred time horizon for viewing data, as shown in Figure 3. In the interviews, users stressed the need for flexible temporal perspectives and accessing historical data:

To me it is not really relevant to look back on my stepcount data on a daily or weekly basis. That's not what motivates me. I am motivated to learn about how I can improve my well-being and mood. It doesn't make sense to look at a week of data and say: 'OK, last week I ordered a pizza and that made me feel good', you know?
I can't build on that. So I figured that I needed more data for some more significant insights. (P2).

In contrast, almost instantaneous data was of most relevance to the users. This showcases how the temporal perspective required by the user is highly dependent on when and in what context a reflection activity takes place. Users emphasised that recent data was most actionable and allowed for quick correction:

It [the tracker] notifies me ten minutes before the start of the next hour, it vibrates for a moment and warns me that I haven't moved in a while (...) Then I go grab a coffee or something, which is at the end of the hall, so that I have a short walk again. (P16)

Our interview data also contained cases where a temporal perspective mismatch prompted users to adapt their tracking experience. One of the triathletes in the study supported their tracking application with custom Microsoft Excel sheets to broaden their temporal perspective:

I really just want to be able to look back into my data from half a year ago and see what I did that week. What did my training look like? How did that affect my heart rate? My fatigue? (...) I can see most of it [the data that 


\section{How often do you check your tracker data?}

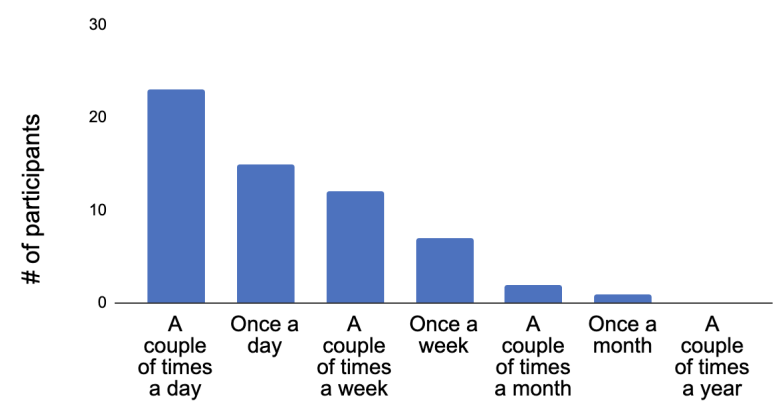

(a)

When you look at your data, what is the time period you look at?

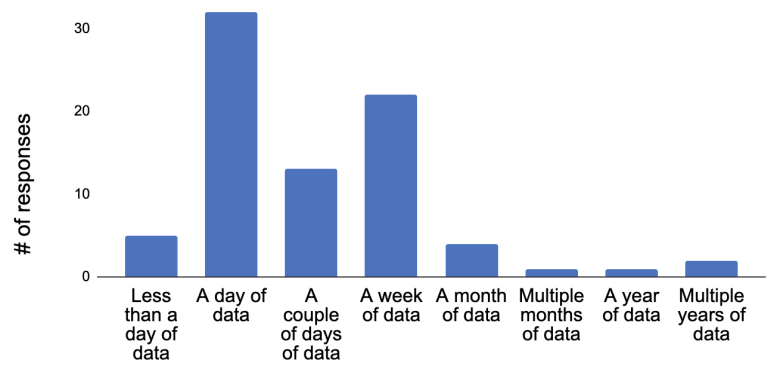

(b)

Figure 3: Data revision frequency (a) and the time period examined (b) by the participants in our survey. Most of the participants frequently (often daily), explored their data. When participants explored their data, they tended to look at short- to medium-term data, as shown by Figure $3 b$. Only $5 \%$ of our participants explored their long-term data.

I need] directly on my watch, but I also use [my own] Excel [spreadsheet]. (P7)

5.2.2 Frequency. Our study showed that users' preferences changed in terms of when they found time for reflection. We found that users were split into those who saw reflecting on data as a primarily spontaneous activity and those who believed that planning was a necessary element of reflection. This was evident in the survey results, which showed that reflecting on tracker data was a mix of spontaneity and planning, see Figure 4.

The need for an individualised reflection schedule which reflected the users' daily routines and their goals in tracking was strongly present in the interviews. One user remarked on how the mismatch between the default notification settings of the tracker and their desired routine and the context of their life produced a negative experience:

What was really annoying to me is one of the default settings. The tracker vibrates and beeps after a period of inactivity (...) which was annoying because of the kind of work I do. While teaching a class, my watch started to vibrate and beep (...) That drove me nuts, so I turned it off. (P6)

However, frequency mismatches were not only experienced as mistimed notifications. Users also reported that long-term reports needed to be presented at times which enabled reflection. Even when users were satisfied with a given report, its temporal perspective and conceptual framing, a report delivered at the wrong time was a barrier for reflection.

There is also a monthly report. And that report really shows what effect each activity and routine has on your sleep and recovery (...) These monthly reports help me to see how things [interventions] I've tried affect me. The overview helps me to look back and reflect (...) WHOOP sends these [monthly reports] through e-mail, [yet] these e-mails come at the most inconvenient times, I don't always have time for that. (P10)

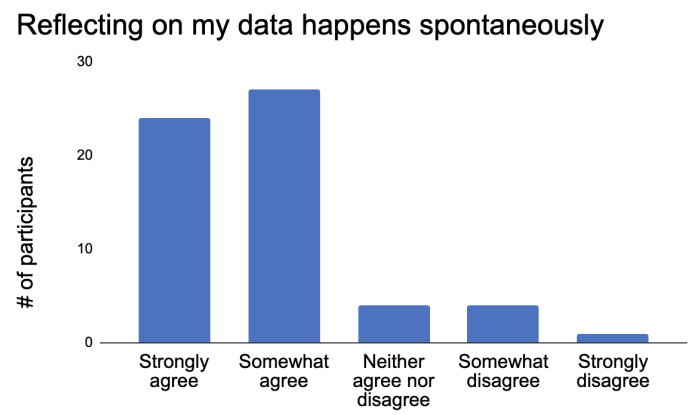

(a)

I plan when to look back on my data.

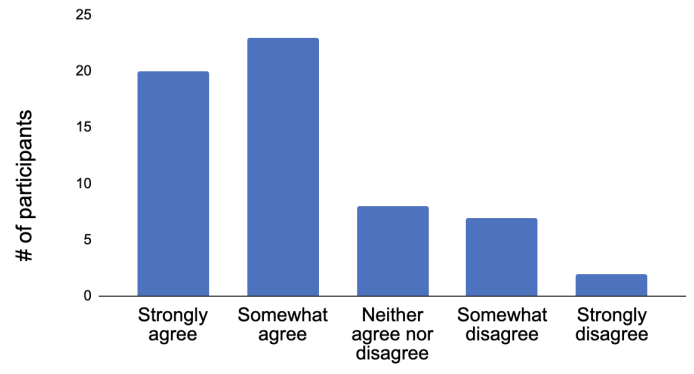

(b)

Figure 4: Reports of spontaneous (a) and planned (b) reflection. Participants both reflected on their data in a spontaneous manner and planned for moments to look back on their data. Reflecting on tracker data is therefore a mix of spontaneity and planning.

\subsection{Conceptual Cycle}

The participants in our study reported that their understanding of their tracker data changed over time. This was apparent in how one of the participants described how their tracker contributed to increased body awareness. Here, the user ascribed predictive 


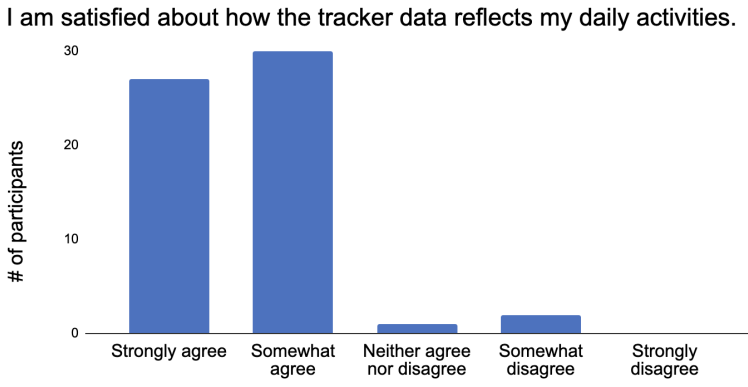

Figure 5: Perception of how tracker data describes activity. Ninety-five per cent of the survey participants reported that they 'strongly agree' or 'agree' with the sentence 'I am satisfied about how the tracker data reflects my daily activities'.

powers to their tracker as it provided data at the desired level of detail:

The data the tracker offers is just incredible. In combination with that band [heart rate band] it even gives me data regarding the length of my steps while running, the height of my foot compared to the ground, in what angle my body is moving. That's just great to me because it gives me information that is usable for preventing injuries for instance. (P6)

Survey data also showed that the accuracy of tracker data describing one's activity was a key criterion. The participants of the survey, the majority of whom were satisfied or very satisfied with their tracking experience, perceived their data as describing their activity well as illustrated in Figure 5.

In contrast, users who experienced a conceptual mismatch struggled to achieve a satisfying reflection experience. There were multiple reasons for facing a conceptual mismatch. One participant discussed how they lacked the necessary knowledge and context to effectively interpret the data provided by their tracker. While the participant did consciously attempt reflection-in-action, she was unable to reflect effectively, and could not relate the highly granular heart rate data to her low-level concept of running intensity.

I don't know what the data [heart rate zones while running] mean exactly. How I should make sense of it. So, I'm not sure when I need to take it a bit more easy [while running] or if I should stay in that zone because it might be beneficial for my muscles or stamina. So yes, it is a reflection attempt, but without a real result. (P13)

Another form of conceptual mismatch was unsuccessfully linking insights from tracker data to more abstract concepts. This was often manifested by the users' declared intent to monitor wellbeing in general and not finding clear means of assessing wellbeing through the tracker. One participant wondered how to relate the number of steps taken (low level of abstraction) to a measure of general fitness (high level of abstraction). The tracker is thus perceived as being out of context, inhibiting facilitated reflection:

Sometimes I think to myself, what does it [the tracker] actually tell me? What do I see? So when I look at my number of steps, or the number of training sessions in a particular week, what does it tell me? I already knew I was a pretty sporty person. So sometimes it makes me question its usefulness. (P3)

Similarly to the temporal cycle, users attempted to bridge the conceptual gaps left by the tracker in order to better facilitate their reflection through either customising the tracker or using additional tools. A common form of additional action which supported the tracker outside of the environment provided by it was manual tracking. P2 chose their own variables to which they ascribed most meaning:

Everyday I [manually] track additional data. The number of calories I eat, my weight, and many more variables. (P2)

But conceptual (re)alignment also took more advanced forms. In the interviews, users reported developing tailor-made solutions for processing their data so that they could interface with it on their desired level of abstractness. In the survey, $45 \%$ of the participants reported using additional tools to process their tracker data. This was also apparent in the interviews. One participant developed software that automatically collected additional data to achieve the desired level of granularity:

I pull the data from my device and put it in a system I made [programmed] myself. I collect 30 to 60 variables both manually and automatically. (P2)

Reprocessing data was another way of assuring conceptual alignment. Despite the tracker presenting the desired scope of data, users were dissatisfied with the form in which it was presented or did not present the data in the desired context, thus causing a conceptual mismatch. P7 manually re-entered values for their tracker to a specially designed Excel sheet. They then added additional subjectively measured variables to achieve the needed level of overview:

So I keep track of each day, an overview [in a spreadsheet] of my resting heart rate, how I'm feeling in general, that is just a subjective score that I attach to it; and the number of hours of sleep (...) Well, I really just want to be able to look back into my data from half a year ago and see what I did that week. What did my training look like? How did that affect my heart rate? My fatigue? (P7)

Finally, we observed that conceptual mismatch was a reason for users to exit the reflection phase of their personal informatics experience. This highlights the cyclic nature of the TMRM-an initial perception of a conceptual match is most often the reason to select a given technology and a conceptual mismatch is likely to lead to no reflection. This is best illustrated by the story of one of the participants who was dissatisfied with the level of abstraction (data granularity) provided by Fitbit, which led to a temporary abandonment of tracking. The participant later returned to tracking and started using the WHOOP which provided metrics at the desired level:

And so, after a few months of not getting proper results, I said '**** it' and I threw it out (...) Then I got a new trainer and he advised me to purchase a WHOOP, because he thought I was training too much. So I was like, no, no, I don't do fitness trackers anymore. He said, 'it's 
not for tracking fitness, but tracking recovery, you have to approach it differently' (...) So I decided to invest in a WHOOP and after three months it really started to work properly and I started changing very small things in my life because of that data. It [the WHOOP] enables me to optimise. And now I train less, and I have the feeling that I get a lot more results from that. (P10)

\section{THE TMRM IN ACTION}

Here, we illustrate how the TMRM can be applied to interpret users' personal informatics experiences and identify opportunities for improving the design of tracking technologies. To that end, we discuss a full user journey from our interview data in detail. We show how the experiences reported in the interviews map to our model and how our model explains the lived experience of the participant. The journey presented below demonstrates the applicability of the model and shows that it accurately depicts current behaviour, constituting an effective way to describe a wide range of current behaviours.

Our example is P7, who is an amateur triathlete who uses a fitness tracker to keep track of his performance and progress in training sessions and competitions. He has been using fitness trackers for the last ten years. Currently, he uses a Garmin 735XT, which is a tracker specifically designed for triathletes. In general, P7 is satisfied with the tracker's abilities. However, in order to be able to reflect on his data, P7 needs to use additional tools. He created a Microsoft Excel spreadsheet and every day, he manually transfers some of his tracker's data to a sheet, and adds additional context data (subjective scores for fatigue and general well-being) to this sheet.

In terms of the TMRM, this user selected a watch specifically designed for triathletes. In his case there is a partial conceptual match between his concept of activity to be tracked and the tracking functionalities of the tracker. First, P7 wants to track more metrics than his tracker allows (conceptual mismatch). Second, the user desires more flexibility in the time period to be analysed (temporal mismatch-perspective). Because of these mismatches, $\mathrm{P} 7$ decided to manually track additional data (conceptual alignment) in a spreadsheet, allowing him to re-enter and maintain facilitated reflection. The sheet enables the participant to review different time periods in his activity and manually track additional metrics. P7's journey shows how users can use or even develop additional tools for tracking to ensure that their reflection cycle is maintained.

Figure 6 presents an annotated version of the TMRM with P7's tracking history. This illustrates how the TMRM can be used to identify starting points for design interventions in building better trackers. The two mismatches are key to understanding P7's story. The user experienced a temporal mismatch when he could not access data in a long-term perspective in his tracker app. Here, the tracker could have offered more control over the time frame or supported integration with other data analytics tools to better support reflection. For temporal alignment, the user chose Excel as the tool. This shows that trackers can support reflection through offering effective data export tools. In the conceptual cycle, P7 was unable to track all metrics in which he was interested. Here, a more flexible device ecology could have been an effective intervention which would have enabled connecting different trackers. As the user decided to manually collect data, an improved system for reflection could allow him to import the data back to the system or analyse the manually collected data together with information for the tracker. This example shows how the TMRM can be used in a design process to map user journeys.

\section{DISCUSSION}

Here, we summarise our results and identify insights for future systems that support reflection in personal informatics. We hope that the TMRM provides a more thorough understanding of the reflection process. As such, the temporal and conceptual cycle also apply to all forms of reflection support, including solutions that do not feature interactive technologies. Our work is situated in past understandings of user journeys where personal informatics systems are enablers, but the model describes a process that is also relevant in an analogue setting. The TMRM is inclusive and describes users with diverse data practices, from minimal bulletjournal note-takers, to data-driven performance athletes.

We built the TMRM with the intention of using it to better understand users in the user-centred design process. Our model can be particularly effective as an additional tool for empathy in activities such as building personas, understanding what range of behaviour to expect from users for a given system or focusing evaluation on reflection support. Our work indicates that future systems should feature explicit and prominent interface elements that enable the user to manipulate the temporal perspective and change from static data representations to more time-dynamic ones. Further, an improved personal informatics artefact should facilitate viewing data on different levels of abstraction simultaneously and empower the user to effectively choose a perspective at a given time. Finally, rather than asking users for presets and configuring the personal informatics experience once, future systems should feature interfaces that undergo constant change and provoke temporal and conceptual exploration.

\subsection{Systems for Reflection Should Support Temporal and Conceptual Manipulation}

Our model interprets reflection as a cyclic process with intermediate stages. This implies that a system that effectively supports reflection helps the user stay within the reflection cycle. However, from a design point of view, reflection as a cycle constitutes a challenge. The iterative nature of the process implies that the user needs undergo constant changes, which, in turn, requires flexibility from the tracking system. Everyday reflection equals everyday changes in perspective and a constant need for new perspective on data. Consequently, a personal informatics system that enhances reflection should support temporal manipulations and different levels of abstraction of viewing the underlying data. Given that the reflection process is a state of constant flux, designers should give users as much control over the temporal and conceptual cycles as possible to allow for efficient alignment.

\subsection{Navigating Levels of Abstraction Is Key in Tracking Experiences}

Our work shows that users frequently changed levels of abstraction on which they interacted with their tracking data. This process 


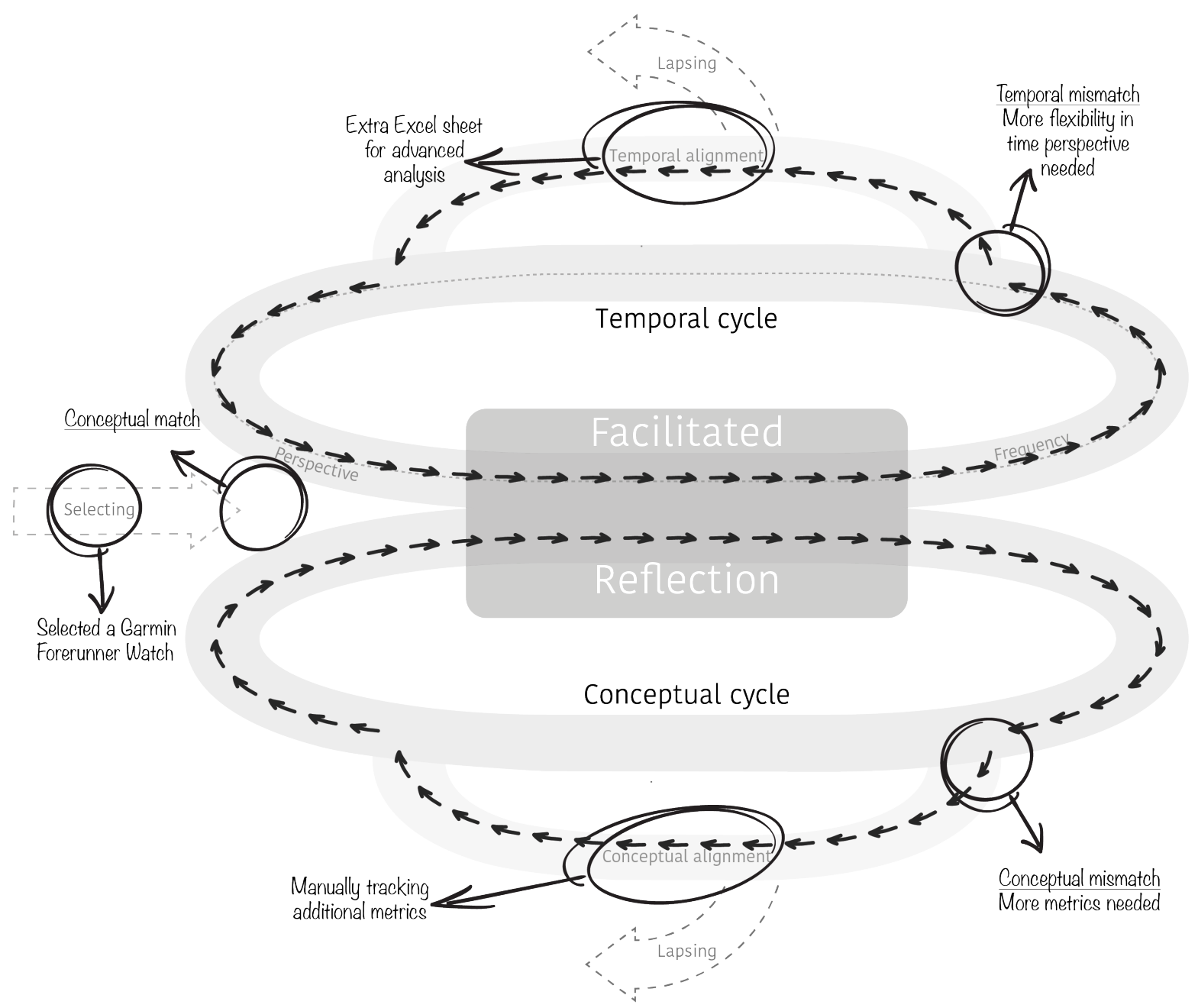

Figure 6: P7's personal informatics journey on the TMRM. Our model help identify key turning points in the user's reflection process. These points show where additional support for reflection is needed.

enables effective reflection as it introduces a change of perspective, viewing situations in a different light [14]. Users reported that frequently changing conceptual perspective was part of their effective reflection routine. Conceptually, this behaviour is explainable by Construal Level Theory [39]. Users approach their fitness data from different psychological distances. The psychological distance can be determined by emotional factors or the context in which data is reviewed. An effective system that supports reflection should be able to offer feedback at different levels of abstraction to match the desired psychological distance thus facilitating conceptual alignment.

The fact that users need flexible levels of abstraction in interacting with their trackers is becoming visible in commercial designs. Recently, many tracker manufacturers introduced metrics that are not directly measured. For instance, Samsung Health [34] does not communicate heart rate variability but a stress score derived from that metric. Garmin sports trackers feature a training efficiency score [15], which is a case of desiring conceptual alignment. The score uses heart rate metrics (low level of abstraction) to build a measure of how effective a training session was (high level of abstraction). Consequently, trackers that effectively support reflection support switching between metrics at different levels of abstraction.

\subsection{Reflection Is an Ever-evolving Process}

Our model proposed conceptualising reflection as a process in a state of constant flux. An intuitive assumption would be for trackers to ensure that users stay in a state of perpetual temporal and conceptual match. However, our study shows that this not only practically impossible, but also possibly harmful for reflection. Participants who reported what we interpreted as mismatches used the mismatch to change the shape of their tracking experience. In practical terms, this process manifested itself in our study by a range of adjustments to the tracker ranging from changing watch faces on the Apple Watch to buying a new tracker. These changes, in turn, offered new perspectives which often resulted in effective reflection. This implies that a system which supports reflection in 
personal informatics does not minimise alignment, but effectively supports it. Users should be made aware of mismatches and presented with the possibility to alleviate them. Here it is important to remember that, in face of a mismatch, users should also be supported in meaningfully abandoning the tracker [11]. Abandonment constitutes a 'conceptual reset', allowing users to possibly re-enter the reflection cycle with a new set of alignment possibilities.

\subsection{The TMRM in the Personal Informatics Landscape}

The TMRM is in line with past conceptual work in personal informatics. Our model features explicit entry and exit points, which relate it to Epstein et al.'s [12] Lived Model of Personal Informatics. The TMRM offers a new perspective on the reflection phase of existing personal informatics models $[12,23]$. The TMRM explains the ways in which users interpret data that lead to discovery and maintenance phases [24] and identifies facilitating factors that help retain maintenance. Further, the TMRM's temporal and conceptual perspective can be used to conceptualise the qualities of personal informatics systems in relation to Epstein et al.'s model, especially in understanding the motives behind decisions in the selection phase.

Further, the TMRM explains parts of Niess and Wozniak's [28] Goal Evolution Model in more detail. In their work, the reflection phase was important in allowing goals to evolve. Our detailed model for reflection suggests that goals are indeed a part of the reflection process and the cyclic nature of reflection supports goal changes. Further, we posit that what Niess and Wozniak called 'goal translation', i.e. moving from qualitative to quantitative goals, is a manifestation of the conceptual alignment on the goal level.

\subsection{Limitations \& Future Work}

We recognise that the framing of the TMRM and the empirical work that led to its construction are affected by certain limitations. First, we note that the participants in our study were all from a Western background. While this is no different from other studies on which this work is based, e.g. [12,27,28], we see a need to broaden the HCI field's understanding of trackers beyond the Western cultural context. We also recruited survey participants using Amazon's Mechanical Turk. While we did require participants to declare that they were using personal tracking technologies, we were unable to verify if that was the case. However, we believe that our approach, i.e. using two differently recruited samples in the two studies offers a robust sample.

Moreover, we note that our inquiry focused on users of fitness trackers. While our model of reflection does not explicitly support only physical activity as a tracked quality, we do not know how it generalises to other domains of personal informatics. In future research, we plan to study reflection across tracking domains. Finally, as reflection is an intimate process, we cannot underestimate the role of personality in reflection, especially as our model is inspired by theories of psychological distance. Grant et al. [17] established that the propensity to reflect is a personality feature. Future work should examine how personality moderates technology-mediated reflection. Also, our model offers the possibility to inspire innovative personal informatics systems [10]. Such novel technologies could then, in turn, be used to further validate the TMRM.

\section{CONCLUSION}

In this paper, we presented the Technology-Mediated Reflection Model (TMRM), which describes practices around reflection for users of fitness trackers. We conducted 20 interviews with active fitness tracker users and an online survey. We constructed the model based on the data obtained in the two studies. Our model interprets reflection as a cyclic process where users constantly seek conceptual and temporal alignment. We showed how the TMRM effectively described user journeys and how it informs the design of systems that support reflection. We hope that our work contributes to a better understanding of fitness trackers and allows more users to improve their well-being.

\section{ACKNOWLEDGMENTS}

This research was financially supported by Utrecht University's Focus Area: Sports and Society. We acknowledge the support of the Leibniz ScienceCampus Bremen Digital Public Health (lsc-diph.de), which is jointly funded by the Leibniz Association (W4/2018), the Federal State of Bremen and the Leibniz Institute for Prevention Research and Epidemiology-BIPS.

\section{REFERENCES}

[1] Amid Ayobi, Paul Marshall, and Anna L Cox. 2020. Trackly : A Customisable and Pictorial Self-Tracking App to Support Agency in Multiple Sclerosis Self-Care. Proceedings of the SIGCHI Conference on Human Factors in Computing Systems (2020), 1-15.

[2] Eric P.S. Baumer, Vera Khovanskaya, Mark Matthews, Lindsay Reynolds, Victoria Schwanda Sosik, and Geri Gay. 2014. Reviewing reflection: On the use of reflection in interactive system design. Proceedings of the Conference on Designing Interactive Systems: Processes, Practices, Methods, and Techniques, DIS (2014), 93-102. https://doi.org/10.1145/2598510.2598598

[3] Eric P S Baumer. 2015. Reflective Informatics: Conceptual Dimensions for Designing Technologies of Reflection. In Proceedings of the 33rd Annual ACM Conference on Human Factors in Computing Systems (CHI '15). Association for Computing Machinery, New York, NY, USA, 585-594. https://doi.org/10.1145/2702123.2702234

[4] Ann Blandford, Dominic Furniss, and Stephann Makri. 2016. Qualitative HCI Research: Going Behind the Scenes. Synthesis Lectures on Human-Centered Informatics 9 (2016), 1-115. https://doi.org/10.2200/S00706ED1V01Y201602HCI034

[5] Patrick Carrington, Kevin Chang, Helena Mentis, and Amy Hurst. 2015. "But, I don't take steps": Examining the inaccessibility of fitness trackers for wheelchair athletes. In ASSETS 2015 - Proceedings of the 17th International ACM SIGACCESS Conference on Computers and Accessibility. 193-201. https://doi.org/10.1145/ 2700648.2809845

[6] Eun Kyoung Choe, Bongshin Lee, Matthew Kay, Wanda Pratt, and Julie A. Kientz. 2015. SleepTight: Low-burden, self-monitoring technology for capturing and reflecting on sleep behaviors. UbiComp 2015 - Proceedings of the 2015 ACM International foint Conference on Pervasive and Ubiquitous Computing (2015), 121-132. https://doi.org/10.1145/2750858.2804266

[7] Eun Kyoung Choe, Bongshin Lee, Haining Zhu, and Nathalie Henry Riche. 2017. Understanding self-reflection: How people reflect on personal data through visual data exploration. In ACM International Conference Proceeding Series (PervasiveHealth '17). Association for Computing Machinery, New York, NY, USA, 173-182. https://doi.org/10.1145/3154862.3154881

[8] Eun Kyoung Choe, Nicole B. Lee, Bongshin Lee, Wanda Pratt, and Julie A. Kientz. 2014. Understanding quantified-selfers' practices in collecting and exploring personal data. Conference on Human Factors in Computing Systems - Proceedings (2014), 1143-1152. https://doi.org/10.1145/2556288.2557372

[9] Sunny Consolvo, Predrag Klasnja, David W. McDonald, and James A. Landay. 2012. Designing for healthy lifestyles: Design considerations for mobile technologies to encourage consumer health and wellness. Foundations and Trends in HumanComputer Interaction 6, 3-4 (2012), 167-315. https://doi.org/10.1561/1100000040

[10] Daniel A Epstein, Clara Caldeira, Mayara Costa Figueiredo, Xi Lu, Lucas M Silva, Lucretia Williams, Jong Ho Lee, Qingyang Li, Simran Ahuja, Qiuer Chen, 
Payam Dowlatyari, Craig Hilby, Sazeda Sultana, Elizabeth V Eikey, and Yunan Chen. 2020. Mapping and Taking Stock of the Personal Informatics Literature. Proc. ACM Interact. Mob. Wearable Ubiquitous Technol. 4, 4 (12 2020). https: //doi.org/10.1145/3432231

[11] Daniel A. Epstein, Monica Caraway, Chuck Johnston, An Ping, James Fogarty, and Sean A. Munson. 2016. Beyond Abandonment to Next Steps: Understanding and Designing for Life after Personal Informatics Tool Use. Proc SIGCHI Conf Hum Factor Comput Syst 165, 7 (2016), 1109-1114. https://doi.org/10.1016/j.cell. 2016.05.007.Mapping

[12] Daniel A. Epstein, An Ping, James Fogarty, and Sean A. Munson. 2015. A lived informatics model of personal informatics. UbiComp 2015 - Proceedings of the 2015 ACM International foint Conference on Pervasive and Ubiquitous Computing (2015), 731-742. https://doi.org/10.1145/2750858.2804250

[13] Clayton Feustel, Shyamak Aggarwal, Bongshin Lee, and Lauren Wilcox. 2018 People Like Me: Designing for Reflection on Aggregate Cohort Data in Personal Informatics Systems. Proceedings of the ACM on Interactive, Mobile, Wearable and Ubiquitous Technologies 2, 3 (2018), 1-21. https://doi.org/10.1145/3264917

[14] Rowanne Fleck and Geraldine Fitzpatrick. 2010. Reflecting on reflection: Framing a design landscape. ACM International Conference Proceeding Series (2010), 216223. https://doi.org/10.1145/1952222.1952269

[15] Garmin. [n.d.]. Forerunner 935 - About Training Effect. https //www8.garmin.com/manuals/webhelp/forerunner935/EN-US/GUID7275629E-743A-4658-A284-C84F42A66AE5.html

[16] Rúben Gouveia, Evangelos Karapanos, and Marc Hassenzahl. 2018. Activity tracking in vivo. In Conference on Human Factors in Computing Systems - Proceedings, Vol. 2018-April. https://doi.org/10.1145/3173574.3173936

[17] Anthony M Grant, John Franklin, and Peter Langford. 2002. The Self-Reflection and Insight Scale: A new measure of private self-consciousness. Social Behavior and Personality 30, 8 (2002), 821-836. https://doi.org/10.2224/sbp.2002.30.8.821

[18] Jonathan Gutman. 1982. A Means-End Chain Model Based on Consumer Categorization Processes. Fournal of Marketing 46, 2 (1982), 60. https://doi.org/10.2307/ 3203341

[19] Ellen Isaacs, Artie Konrad, Alan Walendowski, Thomas Lennig, Victoria Hollis, and Steve Whittaker. 2013. Echoes from the past: How technology mediated reflection improves well-being. Conference on Human Factors in Computing Systems - Proceedings (2013), 1071-1080. https://doi.org/10.1145/2470654.2466137

[20] Malte F. Jung, Nik Martelaro, Clifford Nass, and Halsey Hoster. 2014. Participatory materials: Having a reflective conversation with an artifact in the making. Proceedings of the Conference on Designing Interactive Systems: Processes, Practices, Methods, and Techniques, DIS (2014), 25-34. https://doi.org/10.1145/2598510.2598591

[21] Rafal Kocielnik, Daniel Avrahami, Jennifer Marlow, Di Lu, and Gary Hsieh. 2018 Designing for workplace reflection: A chat and voice-based conversational agent DIS 2018 - Proceedings of the 2018 Designing Interactive Systems Conference (2018), 881-894. https://doi.org/10.1145/3196709.3196784

[22] Rafal Kocielnik, Lillian Xiao, Daniel Avrahami, and Gary Hsieh. 2018. Reflection Companion: A Conversational System for Engaging Users in Reflection on Physical Activity. Proc. ACM Interact. Mob. Wearable Ubiquitous Technol. 2,2 (7 2018). https://doi.org/10.1145/3214273

[23] Ian Li, Anind Dey, and Jodi Forlizzi. 2010. A Stage-Based Model of Personal Informatics Systems. In Proceedings of the SIGCHI Conference on Human Factors in Computing Systems (CHI '10). Association for Computing Machinery, New York, NY, USA, 557-566. https://doi.org/10.1145/1753326.1753409

[24] Ian Li, Anind K Dey, and Jodi Forlizzi. 2011. Understanding My Data, Myself: Supporting Self-Reflection with Ubicomp Technologies. In Proceedings of the 13th
International Conference on Ubiquitous Computing (UbiComp '11). Association for Computing Machinery, New York, NY, USA, 405-414. https://doi.org/10.1145/ 2030112.2030166

[25] Ine Mols, Elise Van Den Hoven, and Berry Eggen. 2016. Informing design for reflection: An overview of current everyday practices. ACM International Conference Proceeding Series 23-27-Octo (2016). https://doi.org/10.1145/2971485.2971494

[26] Jennifer Moon. 1999. Reflection in learning \& professional development. RoutledgeFalmer, New York, NY, USA. 229 pages. https://doi.org/10.4324/9780203822296

[27] Elizabeth L. Murnane, Tara G. Walker, Beck Tench, Stephen Voida, and Jaime Snyder. 2018. Personal informatics in interpersonal contexts: Towards the design of technology that supports the social ecologies of long-term mental health management. Proceedings of the ACM on Human-Computer Interaction 2, CSCW (11 2018). https://doi.org/10.1145/3274396

[28] Jasmin Niess and Paweł W. Woźniak. 2018. Supporting meaningful personal fitness: The tracker goal Evolution Model. Conference on Human Factors in Computing Systems - Proceedings 2018-April (2018), 1-12. https://doi.org/10. 1145/3173574.3173745

[29] Jenny Preece, Yvonne Rogers, Helen Sharp, David Benyon, Simon Holland, and Tom Carey. 1994. Human-Computer Interaction. Addison-Wesley Longman Ltd., GBR.

[30] Shriti Raj, Joyce Lee, Ashley Garrity, and Mark Newman. 2019. Clinical Data in Context: Towards Sensemaking Tools for Interpreting Personal Health Data. Proceedings of the ACM on Interactive, Mobile, Wearable and Ubiquitous Technologies 3, 1 (2019), 1-20. https://doi.org/10.1145/3314409

[31] Wolfgang Reitberger, Wolfgang Spreicer, and Geraldine Fitzpatrick. 2014. Nutriflect: Reflecting collective shopping behavior and nutrition. Conference on Human Factors in Computing Systems - Proceedings (2014), 3309-3318. https: //doi.org/10.1145/2556288.2557384

[32] Samara Ruiz, Sven Charleer, Maite Urretavizcaya, Joris Klerkx, Fernández Castro Isabel, and Erik Duval. 2016. Supporting learning by considering emotions: Tracking and visualization. A case study. ACM International Conference Proceeding Series 25-29-Apri (2016), 254-263. https://doi.org/10.1145/2883851.2883888

[33] Johnny Saldaña. 2015. The coding manual for qualitative researchers. Sage.

[34] Samsung. [n.d.]. Measure your stress level with Samsung Health. https: //www.samsung.com/us/support/answer/ANS00080574/

[35] Donald A. Schön. 1983. The Reflective Practitioner. https://doi.org/10.1542/peds. 2005-0209

[36] Petr Slovak, Chris Frauenberger, and Geraldine Fitzpatrick. 2017. Reflective practicum: A framework of sensitising concepts to design for transformative reflection. Conference on Human Factors in Computing Systems - Proceedings 2017-May (2017), 2696-2707. https://doi.org/10.1145/3025453.3025516

[37] Minhyang Suh and Gary Hsieh. 2016. Designing for future behaviors: Understanding the effect of temporal distance on planned behaviors. In Conference on Human Factors in Computing Systems - Proceedings (CHI '16). Association for Computing Machinery, New York, NY, USA, 1084-1096. https: //doi.org/10.1145/2858036.2858591

[38] Lie Ming Tang and Judy Kay. 2017. Harnessing Long Term Physical Activity Data-How Long-term Trackers Use Data and How an Adherence-based Interface Supports New Insights. Proceedings of the ACM on Interactive, Mobile, Wearable and Ubiquitous Technologies 1, 2 (2017), 1-28. https://doi.org/10.1145/3090091

[39] Yaacov Trope and Nira Liberman. 2010. Construal-Level Theory of Psychological Distance. Psychological Review 117, 2 (2010), 440-463. https://doi.org/10.1037/ a0018963 